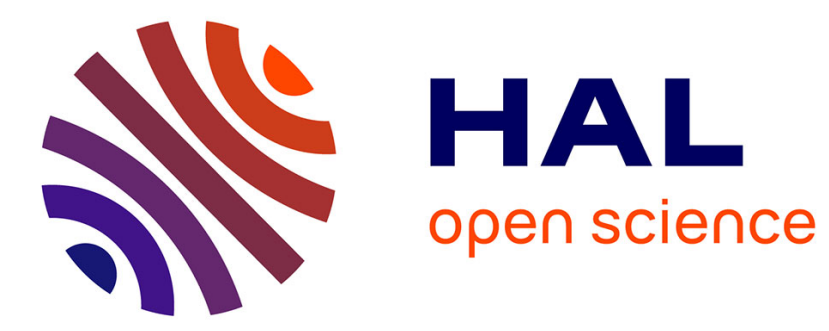

\title{
Quantifying the Mutational Meltdown in Diploid Populations
}

Camille Coron, Sylvie Méléard, Emmanuelle Porcher, Alexandre Robert

\section{To cite this version:}

Camille Coron, Sylvie Méléard, Emmanuelle Porcher, Alexandre Robert. Quantifying the Mutational Meltdown in Diploid Populations. American Naturalist, 2013, 181 (5), pp.623-636. 10.1086/670022 . mnhn-02265203

\section{HAL Id: mnhn-02265203}

\section{https://hal-mnhn.archives-ouvertes.fr/mnhn-02265203}

Submitted on 8 Aug 2019

HAL is a multi-disciplinary open access archive for the deposit and dissemination of scientific research documents, whether they are published or not. The documents may come from teaching and research institutions in France or abroad, or from public or private research centers.
L'archive ouverte pluridisciplinaire HAL, est destinée au dépôt et à la diffusion de documents scientifiques de niveau recherche, publiés ou non, émanant des établissements d'enseignement et de recherche français ou étrangers, des laboratoires publics ou privés. 


\section{CHICAGO JOURNALS}

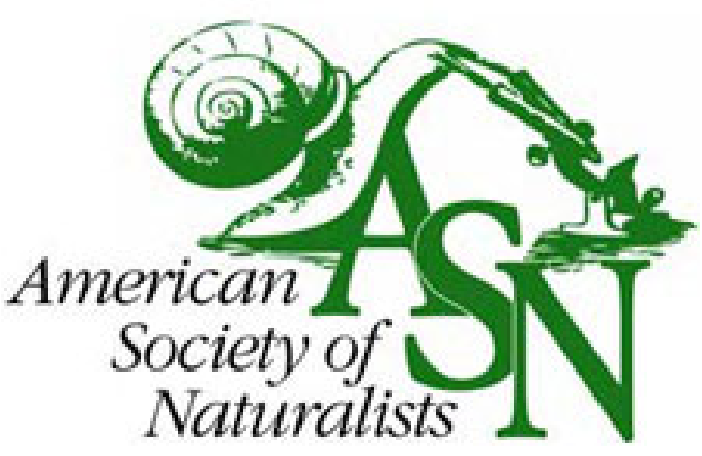

\section{The University of Chicago}

Quantifying the Mutational Meltdown in Diploid Populations.

Author(s): Camille Coron, Sylvie Méléard, Emmanuelle Porcher, and Alexandre Robert

Source: The American Naturalist, Vol. 181, No. 5 (May 2013), pp. 623-636

Published by: The University of Chicago Press for The American Society of Naturalists

Stable URL: http://www.jstor.org/stable/10.1086/670022

Accessed: 18/04/2013 04:50

Your use of the JSTOR archive indicates your acceptance of the Terms \& Conditions of Use, available at

http://www.jstor.org/page/info/about/policies/terms.jsp

JSTOR is a not-for-profit service that helps scholars, researchers, and students discover, use, and build upon a wide range of content in a trusted digital archive. We use information technology and tools to increase productivity and facilitate new forms of scholarship. For more information about JSTOR, please contact support@jstor.org. 


\title{
Quantifying the Mutational Meltdown in Diploid Populations
}

\author{
Camille Coron, ${ }^{1, \star}$ Sylvie Méléard, ${ }^{1}$ Emmanuelle Porcher, ${ }^{2}$ and Alexandre Robert ${ }^{2}$ \\ 1. Centre de Mathématiques Appliquées (CMAP), École Polytechnique, CNRS, Route de Saclay, 91128 Palaiseau Cedex, France; \\ 2. Unité Mixte de Recherche 7204 Muséum National d'Histoire Naturelle-CNRS-Université Pierre et Marie Curie, \\ Conservation des Espèces, Restauration et Suivi des Populations, Muséum National d'Histoire Naturelle, Paris, France
}

Submitted July 25, 2012; Accepted January 10, 2013; Electronically published April 1, 2013

Online enhancements: appendixes.

\begin{abstract}
Aвstract: Mutational meltdown, in which demographic and genetic processes mutually reinforce one another to accelerate the extinction of small populations, has been poorly quantified despite its potential importance in conservation biology. Here we present a model-based framework to study and quantify the mutational meltdown in a finite diploid population that is evolving continuously in time and subject to resource competition. We model slightly deleterious mutations affecting the population demographic parameters and study how the rate of mutation fixation increases as the genetic load increases, a process that we investigate at two timescales: an ecological scale and a mutational scale. Unlike most previous studies, we treat population size as a random process in continuous time. We show that as deleterious mutations accumulate, the decrease in mean population size accelerates with time relative to a null model with a constant mean fixation time. We quantify this mutational meltdown via the change in the mean fixation time after each new mutation fixation, and we show that the meltdown appears less severe than predicted by earlier theoretical work. We also emphasize that mean population size alone can be a misleading index of the risk of population extinction, which could be better evaluated with additional information on demographic parameters.
\end{abstract}

Keywords: population genetics, mutational meltdown, diploid population, logistic birth and death process, fixation probability, mildly deleterious mutations.

\section{Introduction}

Many evolutionary and ecological processes operating in natural populations influence and are influenced by population abundance or density, for example, intraspecific and interspecific competition (Verhulst 1844; Volterra 1931; Lotka 1932), reproduction (Clay and Shaw 1981), trait evolution (Lande 1976; Cherry 1998), and fixation of deleterious mutations (Crow and Kimura 1970, pp. 418-430). These abundance- or density-dependent processes commonly affect population growth, leading to positive (as in the case of cooperation) or negative (as in the case of in-

\footnotetext{
* Corresponding author; e-mail: coron@cmap.polytechnique.fr.
}

Am. Nat. 2013. Vol. 181, pp. 623-636. (C) 2013 by The University of Chicago. 0003-0147/2013/18105-54002\$15.00. All rights reserved.

DOI: $10.1086 / 670022$ traspecific competition) correlations between population growth and abundance/density. Allee effects refer to a positive relationship between population size and the population per capita deterministic growth rate (Stephens and Sutherland 1999) at low density. They limit population viability because the growth rate of populations with an Allee effect becomes negative below a threshold abundance. This phenomenon alone can lead to extinction, but it may also be associated with gradual genetic deterioration, which reinforces the decline in population abundance and results in an extinction vortex (Gilpin and Soule 1986). Mutational meltdown (Lynch and Gabriel 1990) is a particular form of extinction vortex in which demographic and genetic processes mutually reinforce one another. Spontaneous mildly deleterious mutations (Drake et al. 1998; Lynch et al. 1999; Haag-Liautard et al. 2007) can go to fixation and accumulate in small populations, leading to reduced growth rate and reduced population size, which in turn speed up mutation accumulation (Lynch and Gabriel 1990) and can precipitate population extinction.

Besides environmental and demographic threats, genetic considerations are generally addressed in quantitative assessments of endangered species or population viability (Traill et al. 2007), minimum viable population sizes (Shaffer 1981; Gilpin and Soulé 1986), and conservation status (Mace and Purvis 2008). However, the role of genetic deterioration in the extinction process has long been controversial (e.g., Caro and Laurenson 1994; Caughley 1994). In spite of several lines of evidence that endangered species are impacted by genetic factors (Spielman et al. 2004), which may significantly contribute to the risk of extinction in some cases (Saccheri et al. 1998; Blomqvist et al. 2010), the weight of genetic deterioration mechanisms in limiting population viability remains difficult to evaluate and may be strongly variable among and within species (Robert 2011). There is thus a need for more quantitative frameworks to quantify the actual contribution of genetic factors and their interaction with demography to the risk of extinction. In this article, we extend existing qualitative treatments of mutational meltdown (Lynch et al. 1995; Theodorou et al. 2009) 
to (1) gauge the net effect of the actual acceleration of mutation accumulation on population size by comparing our model with a null model, assuming no effect of the current genetic load on the rate of future mutation accumulation, and (2) examine how demographic parameters affect the strength of mutational meltdown. To this end, we introduce a new quantitative approach to analyzing mutational meltdown by computing the probability of fixation of slightly deleterious mutations in diploid populations with stochastic population dynamics. We are specifically interested in the reciprocal interaction between population size and fixation of small-effect deleterious mutations (Lande 1994) in small populations. Unlike most previous studies (e.g., Lynch and Gabriel 1990; Lande 1994), we do not regard population size as a constant parameter but instead build on the pioneering work of Champagnat et al. (2006) and treat population size as a random process in continuous time. The probability distribution of population size depends on individual demographic parameters, which are themselves determined by the genotypes of individuals at a large number of loci subject to recurrent deleterious mutation. Using this model of mutation accumulation in diploid populations with stochastic population dynamics, we assess how the demography-genetics (hereafter demogenetic) feedback accelerates mutation accumulation. Using a null model, we specifically seek to disentangle the relative contribution of two distinct phenomena to extinction risk: (1) the accumulation of deleterious mutations if the mutational meltdown is neglected and (2) the progressive increase in the fixation rate of deleterious mutations through time caused by mutation accumulation and reduced population size, that is, the mutational meltdown per se. We finally highlight that average population size can sometimes be a misleading indicator of the extinction risk and that demographic parameters (birthrates and death rates) may provide useful complementary information. For example, the sensitivity of the rate of mutation fixation to changes in demographic parameters (which themselves depend on the current fixation load) can be used to quantify the extent to which the overall demo-genetic feedback increases the risk of extinction. This allows us to identify situations where the demographic properties of populations might be associated with strong mutational meltdown.

\section{General Model and Ecological Timescale}

\section{General Model}

We consider a population of hermaphroditic, randomly mating diploid organisms following a logistic birth and death process. Mutation to slightly deleterious alleles follows an infinite site model so that each new mutation occurs at a new locus at genomic rate $m_{K}=2 m / K$, where $m$ is the unscaled mutation rate and $K$ is a scale parameter that goes to infinity to model rare mutations, a classical assumption in evolutionary genetic studies (e.g., Lande 1994). We are interested in how the rate of fixation of deleterious mutations changes as mutations accumulate and in the resulting change in demographic parameters, which we model at two timescales: an ecological scale and a mutational scale. At the ecological timescale (i.e., in the limit when $K$ goes to infinity without rescaling of time), a given mutation is lost or fixed before the next occurs. Individuals are hence characterized by their genotype at the mutant locus, with two alleles (wild type and deleterious mutant), and we examine the fate of a single deleterious allele evolving in a genetically homogeneous background (see next section). In contrast, at the longer mutational timescale, new mutations are instantly fixed or lost, and we examine the process of mutation accumulation and subsequent mutational meltdown in a population that is monomorphic at all times (see "The Mutational Timescale").

\section{Evolution of a Single Biallelic Locus at the Ecological Timescale}

At the ecological timescale, we consider a single biallelic locus with a wild-type allele $A$ and a mutant deleterious allele $a$. At this timescale, in the limit when $K$ goes to infinity, as the mutation rate $m_{K}=2 \mathrm{~m} / \mathrm{K}$ goes to 0 , no other mutation occurs before the current mutation is fixed or lost. The population $Z_{t}:=\left(i_{t}, j_{t}, k_{t}\right)$ is then defined at each time $t$ by $i_{t} j_{t}$ and $k_{t}$ the number of individuals with genotypes $A A, A a$, and $a a$, respectively. The process $\left(Z_{t}\right)_{t \geq 0}$ jumps from a point of $\left(\mathbb{Z}_{+}\right)^{3} \backslash\{(0,0,0),(1,0,0),(0,1,0),(0,0,1)\}$ to one of its neighbors at a rate given by the birth- and death rates of each genotype. Individuals are hermaphroditic and selfincompatible, all genotypes have identical density-independent fecundity $b$, and we assume Mendelian reproduction, so that the total birthrates of genotypes $A A, A a$, and $a a$ are

$$
\begin{aligned}
b_{A A}(Z) & :=b\left[\frac{i(i-1)}{N-1}+\frac{i j}{N-1}+\frac{j(j-1)}{4(N-1)}\right], \\
b_{A a}(Z) & :=b\left[\frac{i j}{N-1}+\frac{j(j-1)}{2(N-1)}+\frac{j k}{N-1}+\frac{2 i k}{N-1}\right], \\
b_{a a}(Z) & :=b\left[\frac{k(k-1)}{N-1}+\frac{j k}{N-1}+\frac{j(j-1)}{4(N-1)}\right] .
\end{aligned}
$$

In contrast, per capita death rates are density dependent and combine intrinsic mortality $(d)$ with mortality caused by intraspecific competition (rate $c$ for any pair of individuals). In the absence of competition $(c=0)$, mean individual lifetime is thus $1 / d$ and is decreased for $c>0$. We assume that the deleterious allele $a$ affects only intrinsic 
death rates, such that $d$ is increased by $\delta$ and $\delta^{\prime}$ in the heterozygote and homozygote genotypes, respectively. For $Z=(i, j, k)$ such that $N:=i+j+k \geq 3$, the individual death rates of genotypes $A A, A a$, and $a a$ are thus, respectively,

$$
\begin{aligned}
& d_{A A}(Z):=d+c(N-1), \\
& d_{A a}^{\delta}(Z):=d+\delta+c(N-1), \\
& d_{a a}^{\delta^{\prime}}(Z):=d+\delta^{\prime}+c(N-1),
\end{aligned}
$$

and when $N=2, d_{A A}(Z)=d_{A a}^{\delta}(Z)=d_{a a}^{\delta^{\prime}}(Z)=0$. In principle, the selection parameters $\delta$ and $\delta^{\prime}$ can be of either sign, but here we consider only deleterious mutations (i.e., $\delta^{\prime}>0$; the effect of $\delta$ on fitness is discussed at the end of the section). A key assumption of our model is that no death occurs when only two individuals are left in the population. This prevents extinction but does not hamper our study of the accumulation of deleterious mutations and of the resulting decrease in mean population size. Without extinction, one of the two alleles eventually goes to fixation; we are interested in the probability $u_{i, 1,0}^{\delta, \delta^{\prime}}$ (which also depends on the demographic parameters $b, d$, and $c$ ) that the mutant allele $a$ goes to fixation given an initial population $(i, 1,0)$, that is, a population of $i+1 A A$ individuals in which a single mutation $a$ occurred. More generally, we denote by $u_{i, j, k}^{\delta, \delta^{\prime}}$ the probability that allele $a$ goes to fixation given an initial population $(i, j, k)$. In the neutral case $\left(\delta=\delta^{\prime}=0\right)$, probability theory tells us that $u_{i, j, k}^{0,0}$ is the initial frequency of allele $a$ (see, e.g., Crow and Kimura 1970, p. 425, or Ewens 2004, p. 21): $u_{i, j, k}^{0,0}=(j+2 k) / 2 N$, with $N=i+$ $j+k$. In particular, $u_{i, 1,0}^{0,0}=1 / 2 N=1 / 2(i+1)$. Note that this result does not hold if the population is allowed to become extinct: in this case, there is a nonzero probability that no allele goes to fixation. Assuming weak selection (slightly deleterious effects $\delta$ and $\delta^{\prime}$ ), we can approximate $u_{i, j, k}^{\delta, \delta^{\prime}}$ by its Taylor expansion; that is, we approximate the difference in the probability of fixation between slightly deleterious alleles and neutral alleles by a linear function of $\delta$ and $\delta^{\prime}$. We prove elsewhere that $\left(\delta, \delta^{\prime}\right) \mapsto u_{i, j, k}^{\delta, \delta^{\prime}}$ is differentiable in $(0,0)$ (see Coron 2013) so that we can write

$$
u_{i, j, k}^{\delta, \delta^{\prime}}=\frac{j+2 k}{2 N}-\delta v_{i, j, k}-\delta^{\prime} v_{i, j, k}^{\prime}+o\left(|\delta|+\left|\delta^{\prime}\right|\right),
$$

where $v$ and $v^{\prime}$ are also functions of demographic parameters $b, d$, and $c$. Note that the fixation probability, starting from state $(i, 1,0)$, is then

$$
u_{i, 1,0}^{\delta, \delta^{\prime}}=\frac{1}{2(i+1)}-\delta v_{i, 1,0}-\delta^{\prime} v_{i, 1,0}^{\prime}+o\left(|\delta|+\left|\delta^{\prime}\right|\right) \text {. }
$$

To quantify the strength of selection, we introduce the ratio

$$
\begin{aligned}
\left|\frac{u_{i, 1,0}^{0,0}-u_{i, 1,0}^{\delta, \delta^{\prime}}}{u_{i, 1,0}^{0,0}}\right|= & 2(i+1)\left|\delta v_{i, 1,0}+\delta^{\prime} v_{i, 1,0}^{\prime}\right| \\
& +o\left(|\delta|+\left|\delta^{\prime}\right|\right),
\end{aligned}
$$

that is, the relative difference in the fixation probability of neutral versus deleterious alleles characterized by equation (2). We compute $v_{i, j, k}$ and $v_{i, j, k}^{\prime}$ and study their dependence on the initial population $(i, j, k)$ and on the population demographic parameters $(b, d, c)$ by solving a Dirichlet problem (see app. A, available online, and Coron 2013). Using stochastic calculus, we obtain that $v_{i, j, k}^{\prime}>0$ for every $(i, j, k)$ and that $v_{i, j, k}$ is of the sign of $i-k$. The biological interpretation of the effect of $\delta^{\prime}$ is straightforward since only $a a$ individuals are affected by $\delta^{\prime}: \delta$ being fixed; the larger $\delta^{\prime}$ is, the lower will be the probability of fixation of $a$. The effect of $\delta$ is more intricate because it affects heterozygous individuals, with the same apparent effect on both alleles. In fact, when $A$ is initially the most frequent allele $(i>k)$, allele $a$ is more deleterious when $\delta$ is larger, all else being equal (i.e., $v_{i, j, k}>0$ ); the opposite is true when $a$ is initially the most frequent allele $(k>i)$. In the biologically most relevant cases (i.e., $0<\delta<\delta^{\prime}$ and $i>k$ ), we expect stronger selection as the dominance of $a$ increases. In the particular case of underdominance, starting from a population $(i, 1,0)$, allele $a$ is deleterious if $\delta>0$ and $\delta^{\prime}=0$. These results are consistent with Fisher (1922).

\section{Numerical Results}

Numerical computations show that the fixation probability $u_{N-1,1,0}^{\delta, \delta^{\prime}}$ decreases as the initial population size $N$ increases (fig. 1A), which is consistent with classical results in evolutionary genetics (see, e.g., Ohta 1973). The same figure with a log-log scale can be found in appendix B (fig. B1, available online), showing that the relation between the fixation probability $u_{N-1,1,0}^{\delta, \delta^{\prime}}$ and the initial population size $N$ can be approximated by a power law that depends on $\delta$ and $\delta^{\prime}$. Figure $1 B$ shows that the strength of selection is an increasing function of $N$; this pattern points to the existence of a mutational meltdown, with weaker elimination of deleterious mutations in small populations (see next section).

\section{The Mutational Timescale}

\section{Accumulation of Deleterious Mutations and the Temporal Dynamics of Population Size}

We move to the evolution of a population subject to recurrent deleterious mutations at multiple loci, which, under the assumption of rare mutations, requires a broader timescale. At the mutational scale, we thus scale time $t$ by 

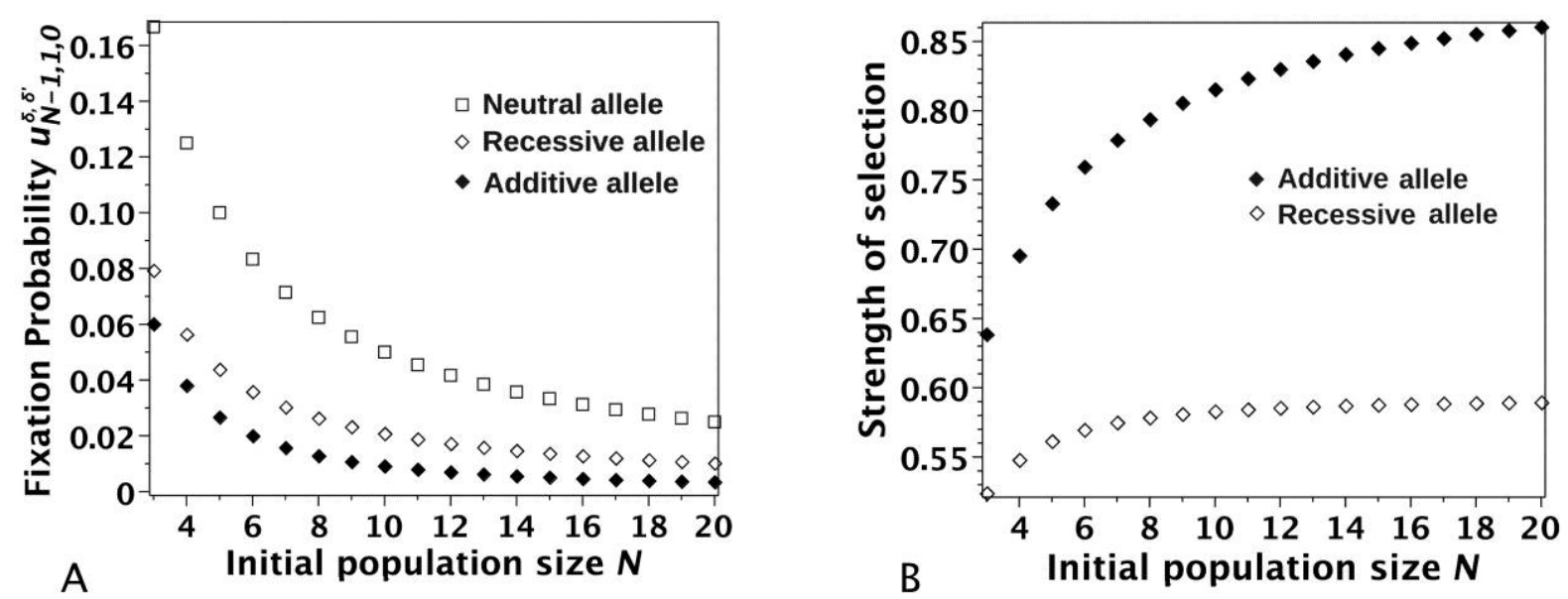

Figure 1: $A$, Fixation probability $u_{N-1,1,0}^{\delta, \delta^{\prime}}$ of an additive deleterious $\left(\delta=\delta^{\prime} / 2\right.$, filled diamonds), recessive deleterious ( $\delta=0$, open diamonds), or neutral $\left(\delta=\delta^{\prime}=0\right.$, squares) allele as a function of the initial population size $N$. B, The strength of selection is measured as the relative difference in fixation probability between a deleterious and a neutral mutation (eq. [4]). In both figures, demographic parameters are $b=10, d=1, c=0.1$, and $\delta^{\prime}=0.2$.

$K$, the parameter we used to model rare mutation (rate $\left.m_{K}=2 m / K\right)$. This allows emergence of new mutations, and we prove, as in the haploid case (Champagnat and Lambert 2007), that when $K$ goes to infinity, new alleles go to fixation or disappear instantaneously due to faster invasion than emergence of mutations (see Coron 2013). Hence, in the limit, an initially monomorphic population stays monomorphic at every time $t$ but with an increasing genetic load. We assume that all mutations have the same effect on intrinsic death rate as described earlier and that individual birthrates and competition rates $b$ and $c$ remain constant. Individuals, therefore, share a common intrinsic death rate $D_{t}$ that changes each time a new mutation gets fixed, and we can therefore track consecutive fixations of deleterious mutations via the change in $D_{t}$. At each time $t$, the population size $N_{t}$ is a random variable following the stationary law of a one-type logistic birth and death process with parameters $b, D_{t}$ and $c$ and with no death when $N=2$. By solving a stationary system (see app. C, available online), we find the probability $p(N, b, d, c):=$ $\mathbb{P}\left(N_{t}=N\right)$ that the population size at time $t$ is equal to $N$ given that $D_{t}=d$ :

$$
p(N, b, d, c):=\frac{\frac{1}{N} \prod_{k=2}^{N-1}[b /(d+k c)]}{\sum_{i=2}^{\infty} \frac{1}{i} \prod_{j=2}^{i-1}[b /(d+j c)]} .
$$

Hence the probability distribution of the population size is directly controlled by its demographic parameters, which contrasts with previous approaches regarding population size as a constant or deterministic parameter (e.g., Crow and Kimura 1970, pp. 5, 7, and 419). Small population size may therefore result from low birthrates or high death rates. An important feature of our model is that the pattern and magnitude of stochastic fluctuations of the size of populations can influence both their long-term effective size and the rate of fixation of deleterious mutations (Wright 1938; Lande 1994).

At the mutational timescale, each new mutation gets either lost, with no effect on the death rate, or fixed instantaneously. In the latter case, the population death rate is increased by $\delta^{\prime}$, since the population changes directly from being monomorphic with type $A A$ to being monomorphic with type $a a$. The stochastic process $\left(D_{t}\right)_{t>0}$ is thus a jump process that jumps from a value $d$ to $d+\delta^{\prime}$ at rate $\tau\left(b, d, c, \delta, \delta^{\prime}\right)$, that is, the rate of fixation of a deleterious mutation, which we compute later (see eq. [6]). When a new deleterious mutation goes to fixation, the stationary law of the population size changes due to an increase in the death rate of all individuals (from $d$ to $d+\delta^{\prime}$ ) that causes a decrease in the mean population size. We can define and compute numerically the expected size of a population with demographic parameters $b, d$, and $c$ :

$$
\bar{N}(b, d, c):=\sum_{N=2}^{\infty} N p(N, b, d, c)
$$

As the death rate $D_{t}$ increases, the mean population size decreases gradually and approaches the minimum value of 2 (see fig. 2 and table $\mathrm{C} 1$ of app. C). Note that when all parameters are multiplied by a constant, the frequency of birth and death events is modified but the distribution of the population size is unaffected (see eq. [5]). In the following, we therefore keep the competition parameter con- 


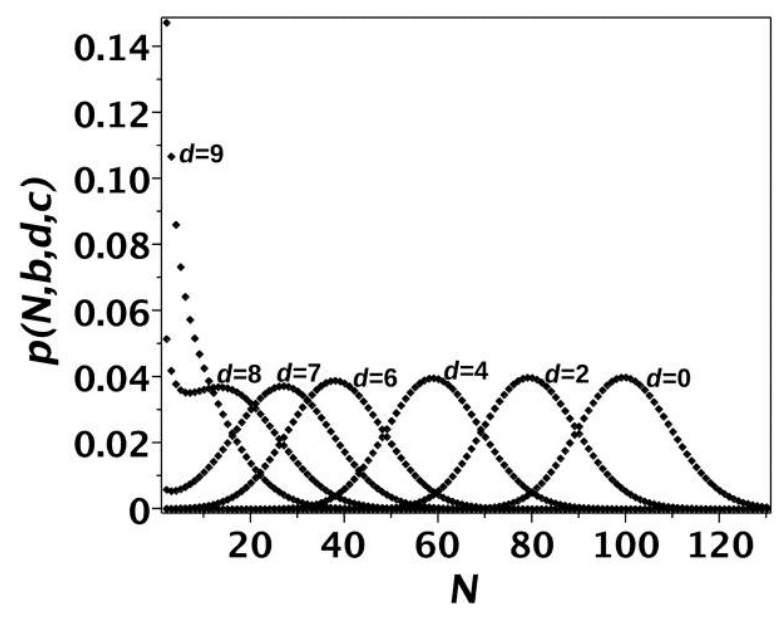

Figure 2: Distribution of the population size under different intrinsic death rates $d$. In this figure, $b=10$ and $c=0.1$.

stant and study the influence of other demographic parameters.

Finally, we examine the temporal dynamics of the mean population size by iterating the effect of mutation fixation on population size. More precisely, in a population with initial demographic parameters $b, d$, and $c$, the mean time to fixation of a deleterious mutation is $T\left(b, d, c, \delta, \delta^{\prime}\right):=$ $1 / \tau\left(b, d, c, \delta, \delta^{\prime}\right)$; at time $t=T\left(b, d, c, \delta, \delta^{\prime}\right)$, we therefore change the intrinsic death rate from $d$ to $d+\delta^{\prime}$ and the mean population size from $\bar{N}(b, d, c)$ to $\bar{N}\left(b, d+\delta^{\prime}, c\right)$. This is repeated through time to obtain the temporal dynamics of population size (see "Quantification of the Mutational Meltdown"). We use these temporal dynamics to evaluate the risk of extinction of a population as the rate of decrease in the mean population size. Here we focused on the arithmetic population mean size, although the harmonic mean is known to be a more accurate index of effective population size in a fluctuating population (Wright 1938). However, the choice of the arithmetic versus harmonic mean did not affect our main results (see "Quantification of the Mutational Meltdown"), and we chose to focus on the simpler arithmetic mean.

\section{The Genetic Load Accelerates the Rate of Fixation of Deleterious Mutations}

Our eventual goals are, first, to prove the existence of a mutational meltdown and, second, to study how demographic parameters influence the strength of this meltdown. In a population of $N$ individuals, the rate of fixation is $2 m N u_{N-1,1,0}^{\delta, \delta^{\prime}}$, which can be averaged over all population sizes $N$ to obtain an overall rate of fixation of deleterious mutations $\tau\left(b, d, c, \delta, \delta^{\prime}\right)$ when the demographic parameters are $b, d$, and $c$ :

$$
\begin{aligned}
\tau\left(b, d, c, \delta, \delta^{\prime}\right):= & 2 m \sum_{N=2}^{+\infty} N u_{N-1,1,0}^{\delta, \delta^{\prime}} p(N, b, d, c) \\
= & m-2 m \sum_{N=2}^{+\infty} N p(N, b, d, c) \\
& \times\left(\delta v_{N-1,1,0}+\delta^{\prime} v_{N-1,1,0}^{\prime}\right)+o\left(|\delta|+\left|\delta^{\prime}\right|\right) .
\end{aligned}
$$

Note here that this fixation rate $\tau$ incorporates both the fixation probability, which is a decreasing function of population size (fig. $1 A$ ), and the rate of mutation $(2 \mathrm{mN})$, which increases with population size. In the particular case of a neutral mutation $\left(\delta=\delta^{\prime}=0\right)$, these two quantities compensate each other so that the fixation rate $\tau$ does not depend on population size or on the demographic parameters $b, d$, and $c$. This is consistent with classical results in evolutionary genetics (see Crow and Kimura 1970, e.g.). For deleterious mutations, we use equation (6) to examine how the mean time to fixation $T$ depends on demographic parameters and dominance relationship among alleles and evolves simultaneously with the mean population size, which yields three important results. First, the mean time to fixation is a decreasing function of the death rate $d$ (figs. 3 and $4 A$ ), which suggests an increase in the rate of fixation of deleterious mutations, that is, a mutational meltdown: fixation of deleterious mutations increases the intrinsic death rate, thereby causing smaller population size and faster fixation of new deleterious alleles, and so on. When

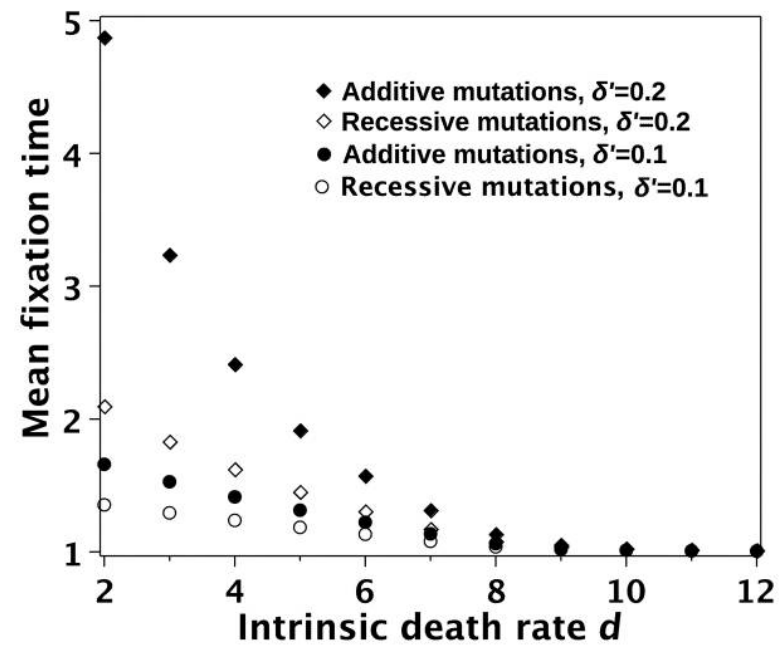

Figure 3: Relationship between $T$, the mean time to fixation of a deleterious mutation, and the population intrinsic death rate $d$ for different selection parameters and dominance cases. Open symbols: recessive mutation $(\delta=0)$; filled symbols: additive mutation $(\delta=$ $\left.\delta^{\prime} / 2\right)$; circles: $\delta^{\prime}=0.1$; diamonds: $\delta^{\prime}=0.2$. Other demographic parameters are $b=10, c=0.1$, and $m=1$. 

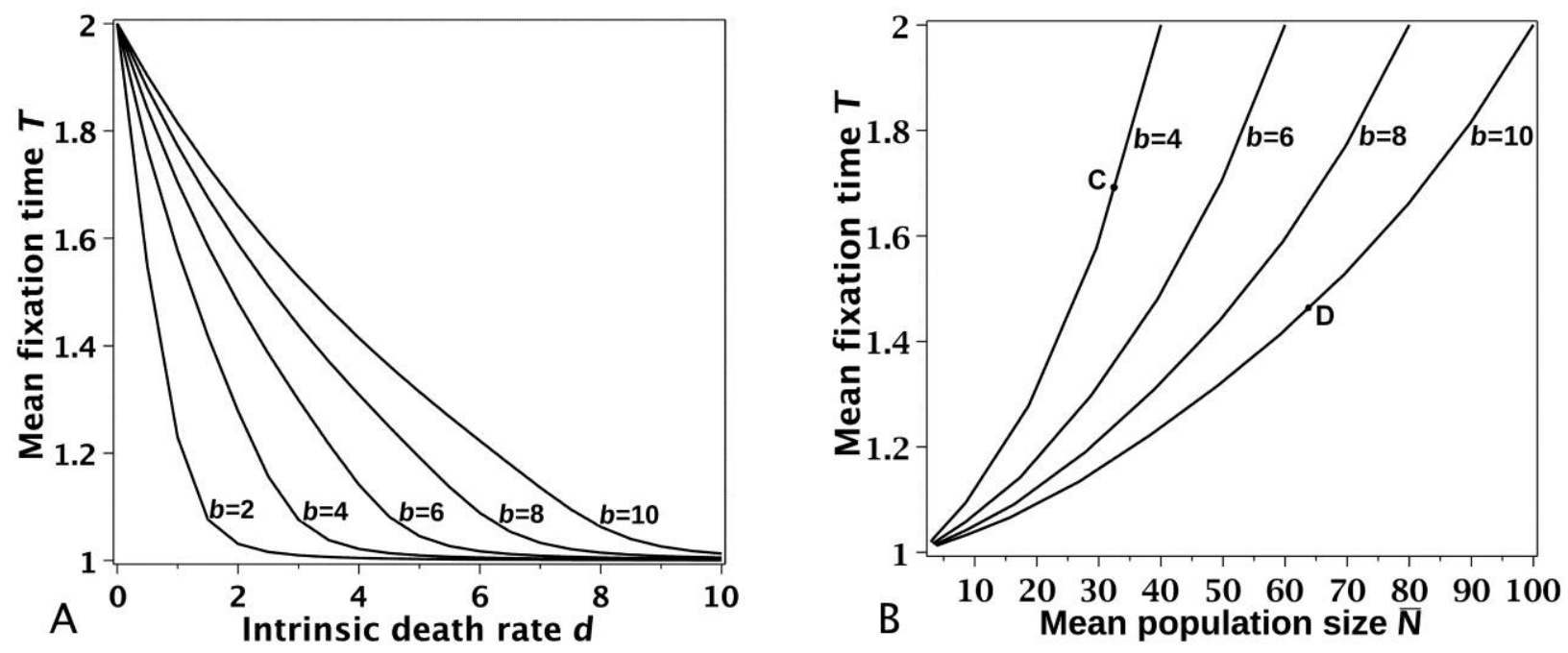

Figure 4: $A$, Relationship between the mean time to fixation of a deleterious mutation $T$ and parameters $b$ and $d$. Each curve corresponds to a fixed value of $b$. Other parameters are $\delta=0.05, \delta^{\prime}=0.1, c=0.1$, and $m=1$. B, Relationship between $T$, the mean time to fixation of a deleterious mutation, and the mean population size $\bar{N}$. Each curve corresponds to a fixed value of $b$ and is obtained by computing the mean population size and mean time to fixation for several values of the natural death parameter $d$. Other parameters are $\delta=0.05$, $\delta^{\prime}=0.1, c=0.1$, and $m=1$. Parameter combinations $C$ and $D$ provide an example in which a larger population size $(D)$ is associated with faster fixation of deleterious mutations.

the intrinsic death rate $d$ becomes large (effectively infinite), the mean fixation time $T\left(b, d, c, \delta, \delta^{\prime}\right)$ converges to the mean fixation time of a neutral mutation $(1 / m$, where $m$ is the unscaled mutation rate) due to a small average population size and dominant effects of drift over selection (for analogous results in other models, see Ohta 1973 and Kimura 1979). As expected, the birthrate $b$ has an opposite effect on the mean fixation time $T\left(b, d, c, \delta, \delta^{\prime}\right)$ (see fig. $4 A$ and app. D, available online), which is an increasing function of $b$ due to higher population sizes, and thus better elimination of deleterious alleles, at higher birthrates. Second, for small values of $\delta$ and $\delta^{\prime}$, the time to fixation of a beneficial mutation with parameters $-\delta$ and $-\delta^{\prime}$ is the symmetrical, with respect to the neutral value $1 / m$, of the time to fixation of a deleterious mutation with parameters $\delta$ and $\delta^{\prime}$. Hence, the mean time to fixation of a beneficial mutation is an increasing function of $d$, implying that the mutational meltdown may also be caused by lower fixation probabilities of beneficial mutations at higher intrinsic death rates. Third, and most importantly, the mean population size provides an incomplete picture of the risk of extinction. More precisely, the mean time to fixation is a generally increasing function of the mean population size $\bar{N}$, as is commonly accepted, but there is no one-to-one correspondence, so two populations with identical mean population sizes can have different extinction risks. Figure $4 B$ provides a symptomatic example, where population $C$ has larger mean time to fixation but smaller population size than population $D$. This phenomenon can be explained by the fact that population size is a random variable and that two populations with identical mean size can have different probability distributions of population size depending on their demographic parameters. The absence of one-to-one correspondence between mean population size and extinction risk is still valid when using the harmonic mean (see next section and app. E, available online). Therefore, unlike existing results from other models (see Crow and Kimura 1970, pp. 345-365), we emphasize the importance of considering not only (arithmetic or harmonic) mean population size but also demographic parameters when studying population extinction risks.

\section{Quantification of the Mutational Meltdown}

Definition of a Null Model. We define mutational meltdown as the acceleration of fixation of deleterious mutations in the population due to previously accumulated mutations (Lynch et al. 1995). Neglecting mutational meltdown is thus equivalent to assuming a constant time to (or rate of) fixation of deleterious mutations. In this case, the extinction risk of a population is simply measured as the decrease in the mean population size caused by this constant rate of fixation. There is an additional net effect of mutational meltdown on extinction that is directly associated with the acceleration of mutation accumulation. Hence, the extinction risk of a population subject to fix- 

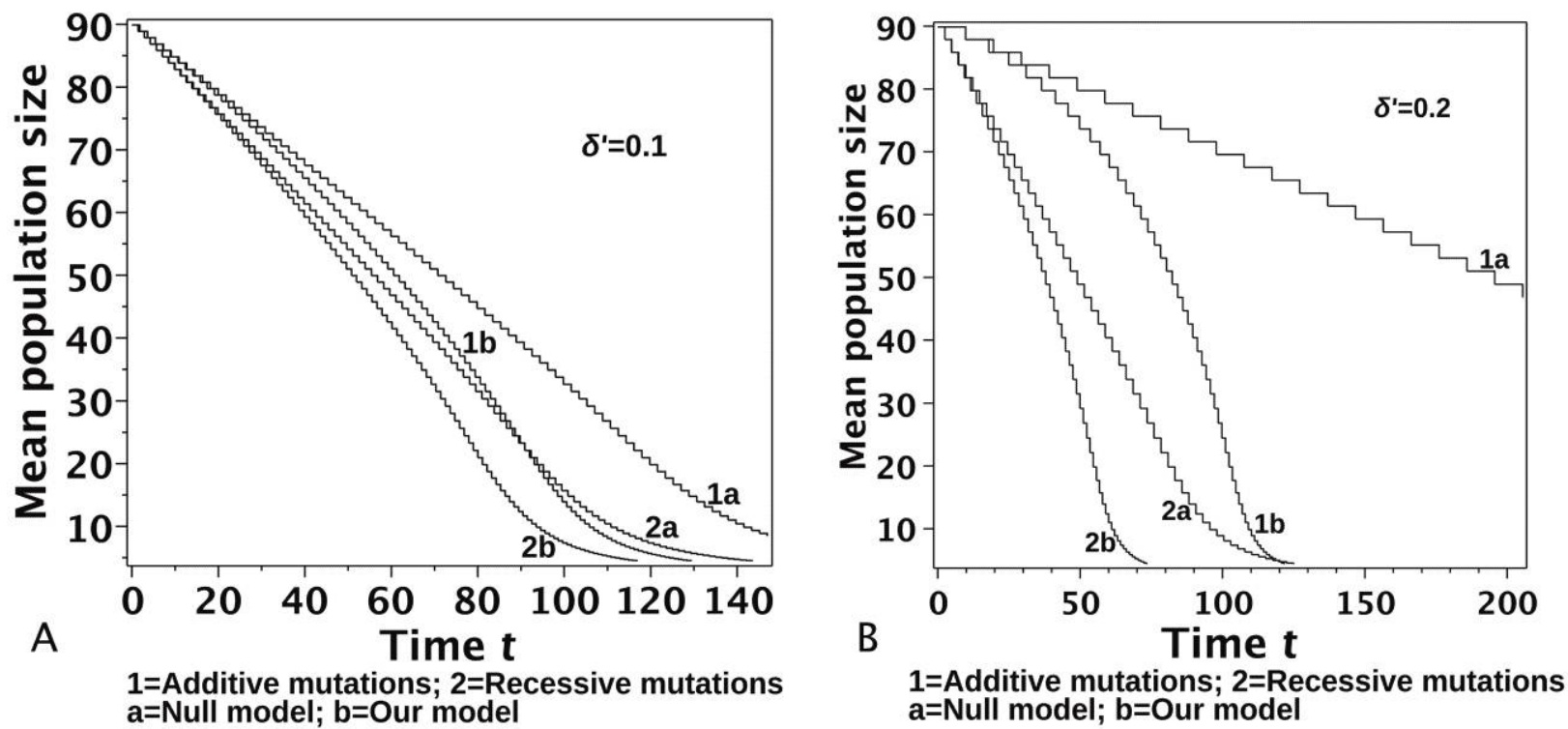

Figure 5: Temporal decrease in the mean population size. In this figure, $b=10, c=0.1, m=1$, and the initial intrinsic death rate is $D_{0}=1$. For $A, \delta^{\prime}=0.1$, whereas for $B, \delta^{\prime}=0.2$. We plot additive $\left(\delta=\delta^{\prime} / 2\right)$ and recessive $(\delta=0)$ cases. For each case, we also plot the temporal dynamics of the mean population size in the corresponding null model. See text ("Accumulation of Deleterious Mutations and the Temporal Dynamics of Population Size") for details on how temporal dynamics were obtained.

ations of deleterious mutations can be divided into two parts: (1) the time to fixation of the first mutation, which provides a good proxy for the extinction risk in the absence of mutational meltdown (constant time to fixation), and (2) the acceleration of fixation between the first and second mutations, which quantifies the contribution of the mutational meltdown to the extinction risk. We are mainly interested in the strength of the mutational meltdown per se, which we measure in two ways. We first compare our model to a null model in which the mutational meltdown is omitted. This null model is the same as our initial model but assumes a constant mean time to fixation, which is equal to the time to fixation of the first mutation. For each set of demographic parameters $b, d$, and $c$, the contribution of the mutational meltdown to the extinction risk is illustrated by the difference in the temporal dynamics of population size in our initial model versus the null model. Second, to eliminate the effect of differences in the time to fixation of the first mutation, we also consider in our model the relative difference between the time to fixation of the first mutation and the time to fixation of the second mutation, that is, the acceleration of fixation:

$$
S\left(b, d, c, \delta, \delta^{\prime}\right):=\frac{T\left(b, d, c, \delta, \delta^{\prime}\right)-T\left(b, d+\delta^{\prime}, c, \delta, \delta^{\prime}\right)}{T\left(b, d, c, \delta, \delta^{\prime}\right)} .
$$

Population Dynamics under a Mutational Meltdown. As deleterious mutations accumulate, the mean population size decreases more and more rapidly relative to the null model with a constant mean fixation time $T\left(b, D_{0}, c, \delta, \delta^{\prime}\right)$ (fig. 5), which is caused by the acceleration of mutation fixations. Note that we obtain similar results when examining the temporal dynamics of the harmonic mean population size (see fig. E1 in app. E), for which the meltdown is slightly stronger than with the arithmetic mean (Wright 1938; Motro and Thompson 1982). As expected, the overall decrease in population size with additive mutations is slower than with recessive mutations, which go to fixation more rapidly. However, with figure 5 only, we cannot compare the influence of the mutational meltdown itself (i.e., the acceleration of mutation fixations) in populations submitted to additive versus recessive deleterious mutations, because these populations have different initial mean fixation times in addition to different strengths of the mutational meltdown. Similarly, although the acceleration in population decline may seem stronger for higher selection coefficient $\delta^{\prime}$ (fig. $5 B)$, this difference is also due to (1) differences in the accelerations of mutation fixations, (2) differences in the initial mean fixation times, and (3) larger influence of mutation fixations on the mean population size for larger $\delta^{\prime}$. From a conservation perspective, it is crucial to quantify the part of the extinction risk that is due to the acceleration of mutation fixations in order to define whether the meltdown can be neglected or not. 

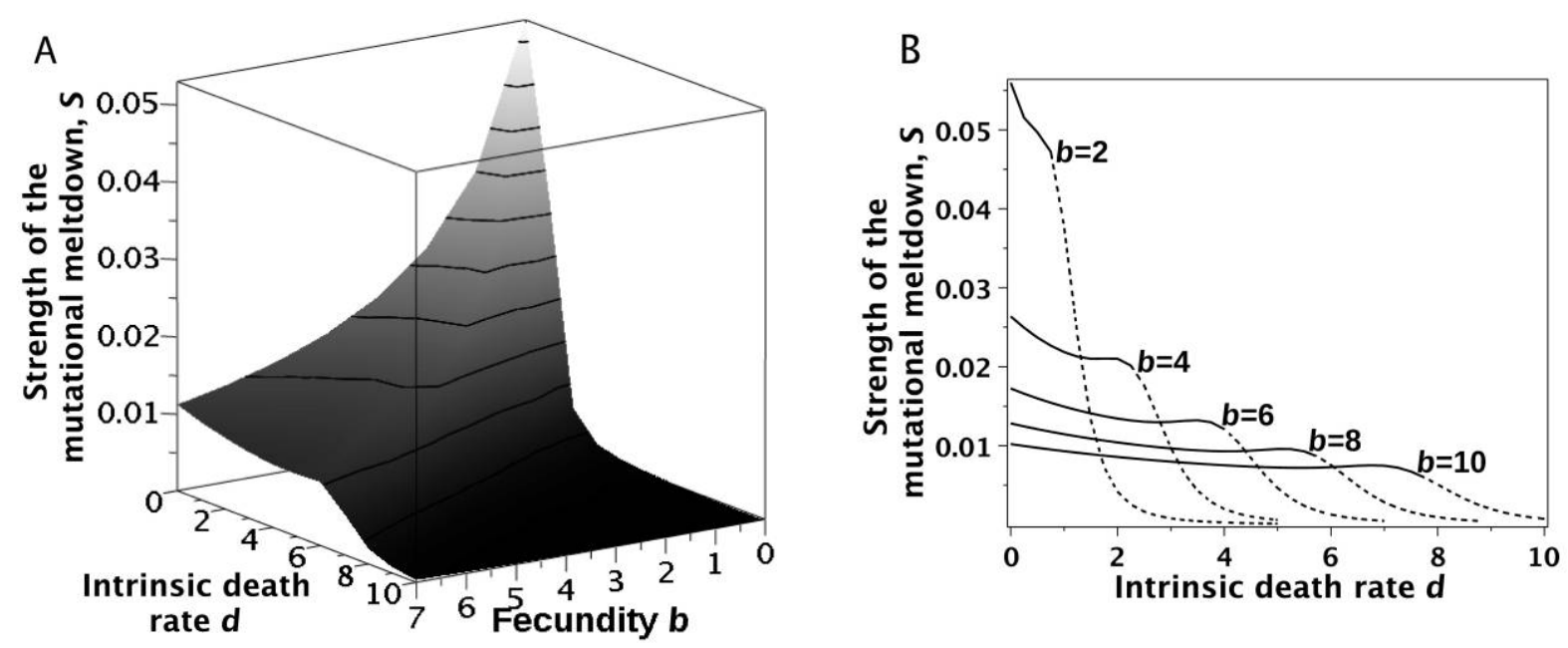

Figure 6: Strength of the mutational meltdown as a function of the demographic parameters $b$ and $d$. A, Solid lines show constant values of the mutational meltdown strength. $B$, Each curve corresponds to a fixed value of $b$ and is dashed above the threshold corresponding to boundary effects (see "Quantifying the Extinction Risk and the Acceleration of Fixations"). In both figures, demographic parameters are $c=0.1, \delta=0.05, \delta^{\prime}=0.1$, and $m=1$.

Quantifying the Extinction Risk and the Acceleration of Fixations. As explained above, we quantify the mutational meltdown using the positive real number

$$
S\left(b, d, c, \delta, \delta^{\prime}\right):=\frac{T\left(b, d, c, \delta, \delta^{\prime}\right)-T\left(b, d+\delta^{\prime}, c, \delta, \delta^{\prime}\right)}{T\left(b, d, c, \delta, \delta^{\prime}\right)},
$$

which is the relative decrease of the mean fixation time between the first and second mutations. $S$ is a function of birth- and death rates (fig. 6) that can be used to define threshold values of the demographic parameters $b$ and $D_{0}$ (initial intrinsic death rate) above or below which the mutational meltdown is small enough to be neglected (fig. $6 A$ ). When fecundity $b$ is fixed, the strength of the mutational meltdown decreases with increasing intrinsic death rate $d$ and goes to 0 as $d$ goes to infinity (fig. 6B): as mutations get fixed, the mean time to fixation of deleterious mutations decreases (i.e., the extinction risk increases), but the population tends to be less subject to mutational meltdown. Note that the right-hand side of curves in figure $6 \mathrm{~B}$ (dashed lines, sharp decrease in the strength of the mutational meltdown above a threshold death rate) can be explained by a boundary effect due to the convergence of the mean population size toward 2 when the intrinsic death rate $d$ increases. These thresholds in $d$ coincide with $d$ values at which a population size of 2 is more probable than a population size of 3 (see fig. 2). These boundary effects are most likely an artifact caused by our assumption of no death when only two individuals are left in the population and should be ignored. However, the general tendency of a decreasing strength of the mutational meltdown with increasing death rates can be observed regardless of boundary effects. As a consequence, the strength of the mutational meltdown under constant fecundity $b$ is an increasing function of the mean population size (fig. 7A), which may appear counterintuitive and inconsistent with previous findings (see Lande 1994). However, when comparing populations with the same initial fixation time (same extinction risk in the null model, i.e., in the absence of mutational meltdown; see fig. $4 A$ ), we show that the strength of the mutational meltdown is a decreasing function of the mean population size (fig. $7 B$ ), such that a threshold mean population size, above which the mutational meltdown is small enough, can still be defined, as in Lande (1994). Importantly, however, this threshold depends on the initial fixation time.

\section{Comparison with Other Models of the Mutational Meltdown}

Our approach is novel in that we combine for the first time in an analytical model two traditions: population genetics approaches, which frequently assume infinite (or, at best, finite but constant) population sizes, and demographic approaches, which are often based on simplified genetic processes. Our results therefore reconcile these two approaches to generate novel results. Below we illustrate this by comparing our results to those obtained with two models that are based on different assumptions regarding population dynamics and genetic architecture and that therefore generate different predictions for the strength of the mutational meltdown: the classical Wright-Fisher pop- 

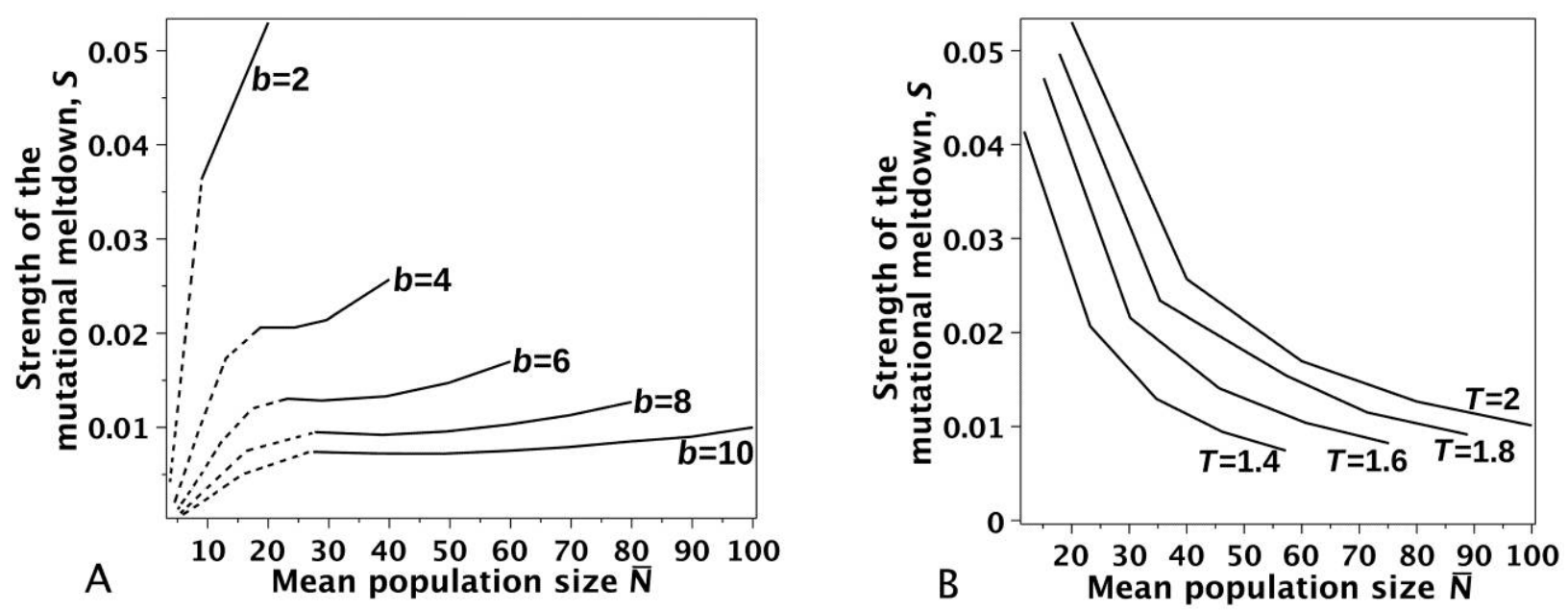

Figure 7: $A$, Relationship between the strength of the mutational meltdown and the mean population size. Each curve corresponds to a fixed value of $b$ and is dashed above the threshold corresponding to boundary effects (see "Quantifying the Extinction Risk and the Acceleration of Fixations"). B, Relationship between the strength of the mutational meltdown and the mean population size for populations with the same initial fixation time $T$, arbitrarily fixed to $1.4,1.6,1.8$, or 2 . For instance, we took $(b, d) \in\{(10,4.2),(8,3.3)$, $(6,2.4)$, $(4,1.6),(2,0.8)\}$ to obtain the curve with $T=1.4$ (see fig. $4 A$ ). In both figures, other demographic parameters are $c=0.1, \delta=0.05$, $\delta^{\prime}=0.1$, and $m=1$.

ulation genetics model and a demo-genetic model of haploid populations.

\section{Comparison with the Wright-Fisher Model}

Crow and Kimura (1970, p. 345) proved that the probability of fixation of a deleterious additive mutation in a population with large, constant size $N$ is

$$
\tilde{u}\left(s, N_{e}, N\right):=\frac{e^{2 s N_{e} / N}-1}{e^{4 s N_{e}}-1}
$$

where $s>0$ is the selection coefficient of a deleterious mutation and $N_{e}$ is the effective population size, which is here a parameter of the model. Using the same timescaling as in our model (see "The Mutational Timescale"), the fixation rate of a deleterious mutation with size $s$ is then equal to $2 \mu N \tilde{u}(s, N, N)$ if the individual mutation rate is $2 \mu$ and $N_{e}=N$. We can use this formula to compare the temporal dynamics of the mean population size in the Wright-Fisher model $\left(N_{e}=N\right)$ versus our model, which requires a modified version of the Wright-Fisher model allowing variable population size. To this aim, we use equation (4) of Clarke (1973) describing the change $\Delta N$ in the population size $N$ by $\Delta N=-2 s N$ at each new fixation. The population size remains constant between two fixations, and we denote by $N_{0}$ the initial population size. To compare the two models, we first set identical initial mean population sizes (i.e., $N_{0}=\bar{N}\left(b, D_{0}, c\right)$, where $b, D_{0}$, and $c$ are the initial demographic parameters in our model). Next, we rescale time and fitness in the Wright-Fisher model so that the first mutation that goes to fixation has the same mean fixation time and the same impact on the mean population size as in our model, that is, $2 \mu N_{0} \tilde{u}\left(s, N_{0}, N_{0}\right)=\tau\left(b, D_{0}, c, \delta, \delta^{\prime}\right)$ and $2 s N_{0}=\bar{N}\left(b, D_{0}, c\right)-\bar{N}\left(b, D_{0}+\delta^{\prime}, c\right)$, with the latter equality giving a relationship between $s$ and $\delta^{\prime}$. We show that the mutational meltdown is stronger in the WrightFisher model (fig. 8), that is, the mean population size collapses more rapidly. This can be understood examining the Taylor expansion of equation (7) when $N_{e}=N$ :

$$
\frac{1}{2 N}\left[1-s N\left(2-\frac{1}{N}\right)\right]+o(s),
$$

which yields a strength of selection of $s N(2-1 / N)$, to be compared with equation (4) and figure $1 B$ of our model; for large population sizes, the strength of selection is equivalent to $2 s N$ in the Wright-Fisher model, whereas it is bounded in our model. As a result, the strength of selection and the time to fixation decrease more rapidly in the WrightFisher model as the population size decreases. This suggests that the mutational meltdown is overestimated with the Wright-Fisher model when compared to our model.

\section{Comparison with the Haploid Model of Champagnat and Lambert}

In a more realistic demographic model, Champagnat and Lambert (2007) studied the fixation of small mutations in a haploid population. Their model is a birth and death 

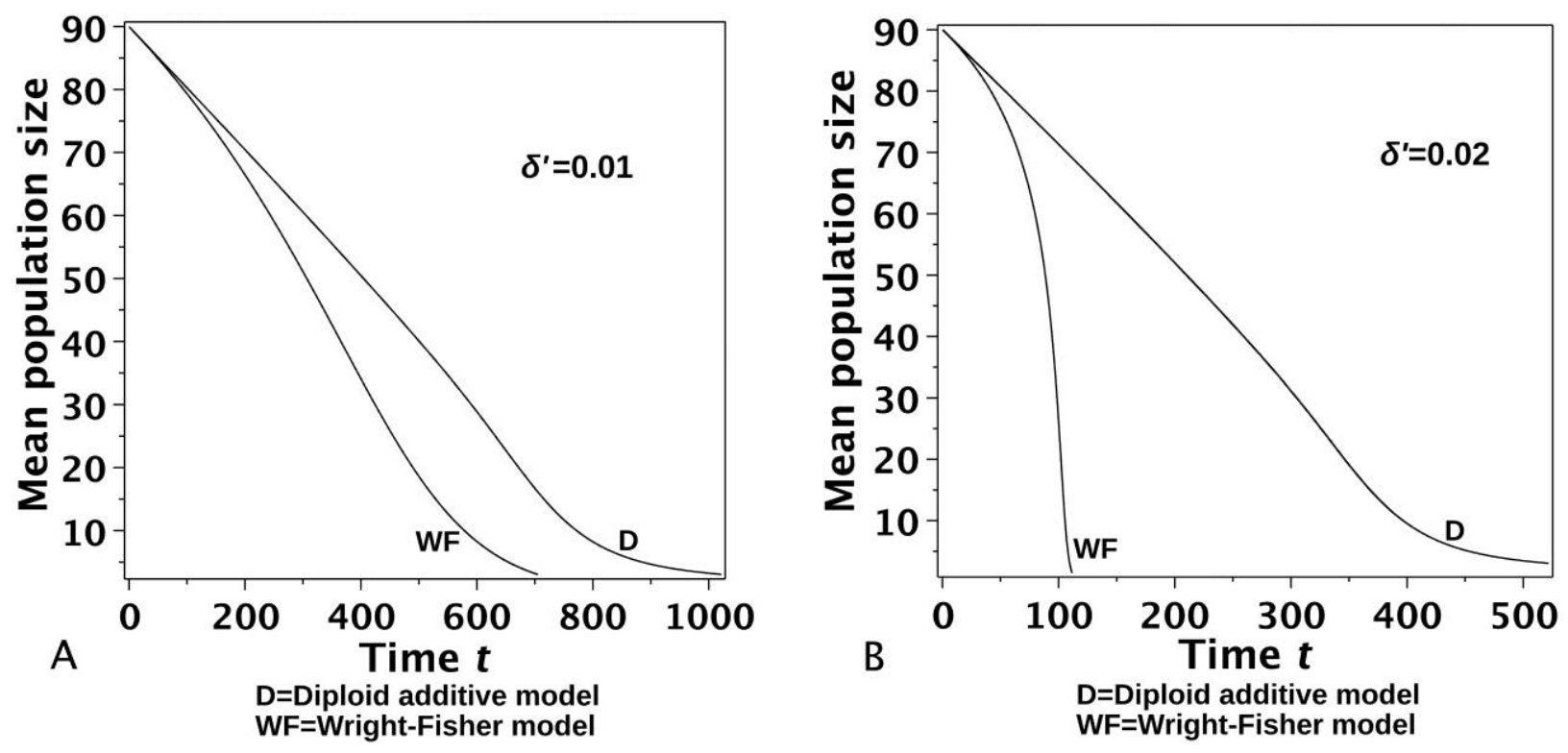

Figure 8: Temporal dynamics of the mean population size in our additive diploid model (D) and the Wright-Fisher model (WF). In these figures, $b=10, c=0.1, m=1$, initial intrinsic death rate is $D_{0}=1$ (model D), and $N_{0}=\bar{N}(10,1,0.1)$ (model WF). For $A, \delta^{\prime}=0.01$, whereas for $B, \delta^{\prime}=0.02$; in both cases, $\delta=\delta^{\prime} / 2$.

process with competition similar to ours but in which birthrates are derived from haploid reproduction rules (i.e., the birthrate of an individual with a given genotype is proportional to the number of individuals with this genotype in the population). Comparing the haploid and diploid populations is, however, not straightforward, particularly because the latter produces twice as many alleles in one birth and contains twice as much genetic material for the same population size. We can nonetheless rely on the expectation that under Hardy-Weinberg equilibrium and large population sizes, a diploid population under additive selection can be approximated by a haploid population. We chose to compare haploid versus diploid models by considering populations with identical rates of fixation of neutral mutations, identical effects of mutations, and identical initial demographic parameters. Under these conditions, the temporal decrease in mean population sizes is in fact comparable in both models when deleterious mutations have additive effects in diploid organisms (fig. $9 A$ ). The only differences appear for really small populations, which is at least partly due to the fact that the minimum diploid population size is 2 , whereas the minimum haploid population size is 1 . Our model, however, allows us to examine nonadditive mutations that cannot be studied in haploid models. This is particularly relevant in diploid organisms, in which most deleterious mutations have partly to fully recessive effects (García-Dorado et al. 2004). We show that the mutational meltdown is stronger with additive mutations than with recessive ones (fig. 9B): its strength increases with the effect of mutations in heterozygotes $\delta$, when $\delta^{\prime}$, the effect of mutations in homozygotes, is kept constant.

\section{Discussion}

In this article, we provided a more quantitative framework for the study of mutational meltdown than previous theoretical treatments and modeled the stochastic dynamics of population size in diploid organisms. As in several previous models, we demonstrated the existence of a mutational meltdown by showing that as deleterious mutations accumulate, the population size decreases more and more rapidly relative to a null model with constant mean fixation time of deleterious mutations. We showed that this fixation time is a decreasing function of the intrinsic death rate, an increasing function of the fecundity, and converges toward the mean fixation time of neutral mutations as the intrinsic death rate goes to infinity. Our approach takes the study of mutational meltdown a step further by demonstrating that mean population size is not always the best indicator of extinction risk for populations subject to fixation of deleterious mutations. We also used our results to suggest a new quantification of the mutational meltdown (i.e., the acceleration of mutation fixations) and to define combinations of demographic parameters for which the mutational meltdown can or cannot be neglected. Finally, our results suggest that the mutational meltdown per se may not be as severe as predicted by earlier pop- 

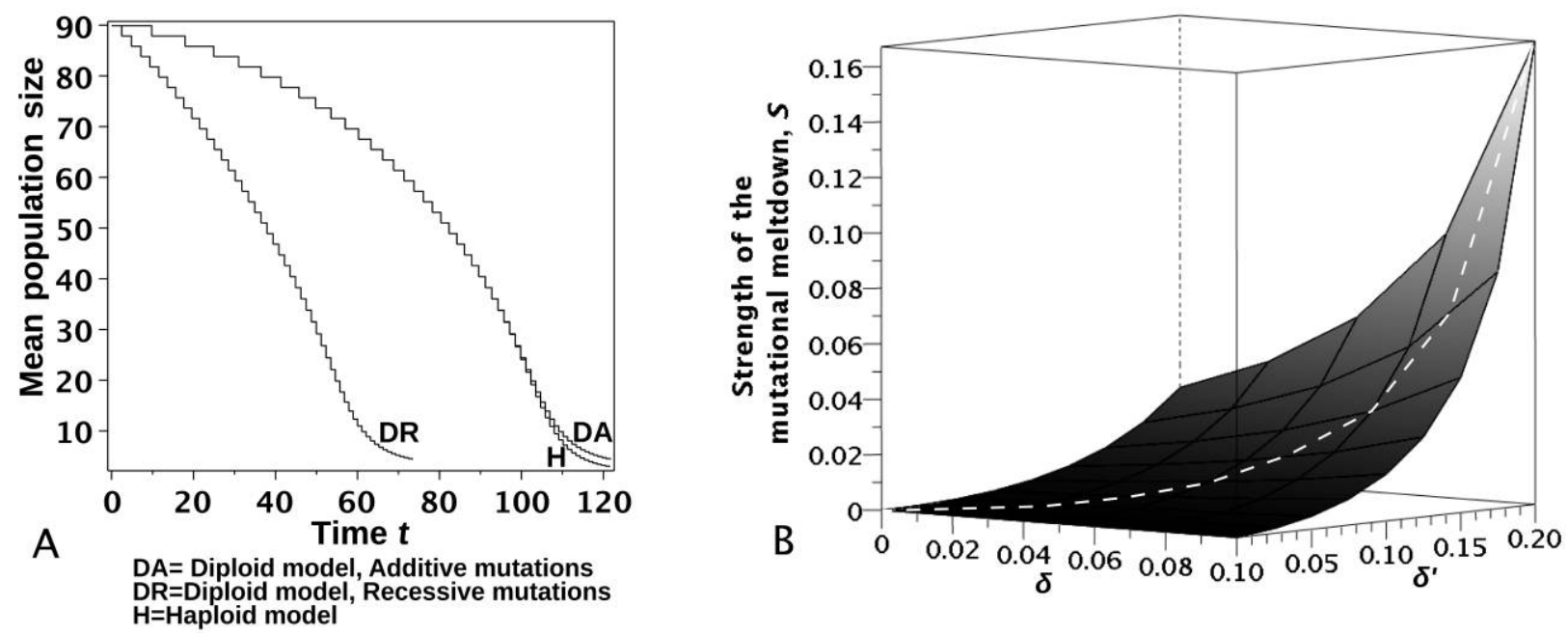

Figure 9: $A$, Temporal dynamics of the mean population size in haploid versus diploid populations. The lower curve DR is reproduced from curve $2 \mathrm{~b}$ of figure $5 \mathrm{~B}$, that is, the temporal dynamics of the mean population size in the recessive case when $\delta=0$. The upper curves are the change in the mean population size with time in a haploid population, $\mathrm{H}$, derived from Champagnat and Lambert (2007), and in a diploid population with additive mutations, DA, reproduced from curve $1 \mathrm{~b}$ of figure $5 B$. Parameter values are $b=10, c=0.1, m=1$, and $\delta^{\prime}=0.2$, and the initial intrinsic death rate is $d=1$. S Strength of the mutational meltdown as a function of the effect of deleterious mutations in the heterozygote $(\delta)$ and in the homozygote $\left(\delta^{\prime}\right)$. The white dashed line corresponds to the additive case $\left(\delta=\delta^{\prime} / 2\right)$. In this figure, $b=10, d=1, c=0.1$, and $m=1$.

ulation genetics models assuming constant population size or haploid models. In the following, we discuss the implications of our results for conservation and the limitations of our approach.

\section{Quantifying the Mutational Meltdown}

While the ultimate causes of most species extinctions are environmental (Brooks et al. 2002), various environmental constraints can have a variety of secondary consequences on ecological and genetic processes. This implies that, depending on the situation faced by populations, the relative weights of nongenetic components (e.g., ecological factors associated with highly variable and/or low average growth rate), genetic components (e.g., inbreeding depression, accumulation of deleterious mutations), and of their interaction (e.g., mutational meltdown in the strict sense) may strongly vary. Here we developed a framework that allows accounting for all three components. Although we focused on quantifying the interaction component (i.e., the demographically mediated effect of the load of fixed mutations on the rate of future mutation fixations), we also provided results on the "pure" genetic component (i.e., the rate of mutation fixation, independent from the load of fixed mutations) and revealed that the extinction risk for populations subject to recurrent deleterious mutation fixations can be quantified both with the initial mean fixation time of a deleterious mutation and with the accel- eration of mutation fixations, which quantifies the strength of the mutational meltdown. We also highlighted that the quantification of the extinction risk in terms of demographic parameters rather than population size could provide a more accurate picture of the population temporal dynamics. Indeed, populations of similar sizes can have different initial times to fixation of deleterious mutations, and therefore different risks of extinction, but can also suffer different strengths of the mutational meltdown. Figure $6 A$ provides values of the demographic parameters for which the mutational meltdown can be neglected or is below a fixed value. However, the mean population size is a more intrinsic demographic characteristic of a population, and to link with previous works on minimum viable size (Shaffer 1981), we observe that in populations with the same extinction risk when neglecting the mutational meltdown (i.e., the same initial mean fixation time), the strength of the mutational meltdown is a decreasing function of the initial mean population size (fig. $7 B$ ). This gives a theoretical definition of a minimum viable population size regarding deleterious mutations accumulation. Finally, our results demonstrate that the magnitude of the mutational meltdown is highly dependent on the underlying demo-genetic model but also on demographic and mutational parameters (e.g., level of dominance). $\mathrm{Nu}$ merical applications indicate, for instance, that genetic models different than ours may strongly overestimate the magnitude of the mutational meltdown (see, e.g., the 
Wright-Fisher model, fig. 8). On the other hand, neglecting the mutational meltdown might lead to strong underestimation of the final/overall speed of mutation accumulation and, subsequently, to overestimation of the time to extinction (fig. $5 B$ ).

\section{Limitations}

One major limitation of our model is that a population cannot become extinct. This creates obvious boundary effects (see, e.g., fig. 6), which preclude an accurate analysis of very small populations ( $<10$ individuals). Our results remain valid if we assume very rare extinction, but even then boundary effects are present. Nonetheless, we are confident that our main results regarding the quantification of the strength of the mutational meltdown are not affected by this assumption because the effects of mutation accumulation are detectable after the fixation of a few deleterious mutations, that is, a long time before extinction for most populations. In the case where extinction probability is relatively high, fixation probabilities (including that of neutral alleles) are modified because the population can become extinct before any allele goes to fixation. This could be accounted for by computing the expected number of mutations that get fixed before the population becomes extinct, but this would require another timescale because the population would instantaneously become extinct at the mutational timescale presented here.

We also assumed no epistasis and free recombination of all loci (no linkage). Population genetic theory (Hill and Robertson 1966; Felsenstein 1974) and empirical results (Betancourt and Presgraves 2002) suggest that the efficacy of natural selection is generally limited by linkage. The consideration of linkage is not expected to qualitatively modify our results regarding the occurrence of the mutational meltdown and the effects of demographic parameters on its magnitude, but it may generate a more detrimental effect of mutation accumulation in all cases. Similarly, synergistic or antagonistic epistasis is likely to influence the strength of selection. In particular, if deleterious mutations interact synergistically, they may be more efficiently removed by selection, which may result in a reduced load (Charlesworth 1990). However, no clear pattern of epistasis (synergistic or antagonistic) is apparent from empirical studies (Elena and Lenski 1997).

\section{Implications and Forthcoming Works}

Our results have critical implications in the field of conservation biology, in which the projected viability of endangered populations is generally derived from demographic models (based on specific demographic components [Beissinger and McCullough 2002]) and/or genetic models (based on population size [Franklin and Frankham 1998]). While mere juxtapositions of these two kinds of estimates might provide reasonable estimates of the extinction risk in some cases (Robert 2011), this and other works (Lynch et al. 1995) indicate that the demo-genetic interaction may strongly affect both the dynamics of mutations (i.e., fixation rate) and population viability. However, proper consideration of this interaction requires the use of advanced concepts and sophisticated tools, which challenges its use as a standard/operational method in conservation. In this context, we hope that our framework will be useful in outlining a conceptual basis to differentiate situations in which the mutational meltdown has minor effects on the risk of extinction from those in which it cannot be neglected. Our choice of considering finite diploid populations of variable sizes and evolving continuously in time was motivated by the urgent need for theoretical models with strong practical implications in the field of biodiversity conservation. Most species of conservation concerns are diploid, iteroparous organisms (Seddon et al. 2005), and endangered populations generally exhibit high year-to-year variance in population size, due to either sampling or process variation (Lande et al. 2003). Our overlapping generations framework allows not only to assume varying population sizes over long timescales but also to relax the assumption of constant population size within generations (as, e.g., in the modified Wright-Fisher model presented above). This is of particular interest to assess the viability of small populations of longlived species (typically, bird and mammal species), in which population sizes may vary by orders of magnitude within a single generation for both intrinsic (e.g., the mutational meltdown itself) or environmental reasons. Further, by incorporating explicit descriptions of birth and death processes, our framework can also incorporate deterministic or stochastic variation in demographic parameters, which would allow us to model environmental stochasticity. An interesting perspective is to compare the weights of environmental stochasticity and deleterious mutation accumulation on the extinction risk of populations (Lande 1993; Spielman et al. 2004).

\section{Acknowledgments}

This work benefited from the support of the ANR (Agence Nationale de la Recherche) Modèles Aléatoires en Écologie, Génétique et Évolution (MANEGE; ANR-09-BLAN-0215) and from the Chair "Modélisation Mathématique et Biodiversité" of Veolia Environnement-École PolytechniqueMNHN-Fondation X. The authors would like to thank anonymous reviewers, Associate Editor P. Taylor, and Editors R. G. Shaw and M. A. McPeek for careful review and 
for providing us with very constructive comments that helped to improve the manuscript.

\section{Literature Cited}

Beissinger, S., and D. E. McCullough. 2002. Population viability analysis. University of Chicago Press, Chicago.

$\rightarrow$ Betancourt, A., and D. Presgraves. 2002. Linkage limits the power of natural selection in Drosophila. Proceedings of the National Academy of Sciences of the USA 99:13616-13620.

$\rightarrow$ Blomqvist, D., A. Pauliny, M. Larsson, and L. Flodin. 2010. Trapped in the extinction vortex? strong genetic effects in a declining ver tebrate population. BMC Evolutionary Biology 10:33.

$\rightarrow$ Brooks, T., R. Mittermeier, C. Mittermeier, G. Da Fonseca, A. Rylands, W. Konstant, P. Flick, et al. 2002. Habitat loss and extinctior in the hotspots of biodiversity. Conservation Biology 16:909-923.

$\rightarrow$ Caro, T., and M. Laurenson. 1994. Ecological and genetic factors in conservation: a cautionary tale. Science 263:485-486.

$\rightarrow$ Caughley, G. 1994. Directions in conservation biology. Journal of Animal Ecology 63:215-244.

$\rightarrow$ Champagnat, N., R. Ferrière, and S. Méléard. 2006. Unifying evolutionary dynamics: from individual stochastic processes to macroscopic models. Theoretical Population Biology 69:297-321.

$\rightarrow$ Champagnat, N., and A. Lambert. 2007. Evolution of discrete populations and the canonical diffusion of adaptive dynamics. Annals of Applied Probability 17:102-155.

$\rightarrow$ Charlesworth, B. 1990. Mutation-selection balance and the evolutionary advantage of sex and recombination. Genetics Research 55:199-221.

Cherry, J. 1998. Should we expect substitution rate to depend on population size? Genetics 150:911-919.

$\rightarrow$ Clarke, B. 1973. The effect of mutation on population size. Nature 242:196-197.

$\rightarrow$ Clay, K., and R. Shaw. 1981. An experimental demonstration of density-dependent reproduction in a natural population of Diamorpha smallii, a rare annual. Oecologia (Berlin) 51:1-6.

Coron, C. 2013. Stochastic modeling of density-dependent diploia populations and extinction vortex. Journal of Applied Probability (forthcoming), arXiv:1207.4920v1.

Crow, J., and M. Kimura. 1970. An introduction to population genetics theory. 2nd ed. Harper \& Row, New York.

$\rightarrow$ Drake, J., B. Charlesworth, D. Charlesworth, and J. Crow. 1998. Rates of spontaneous mutation. Genetics 148:1667-1686.

$\rightarrow$ Elena, S., and R. Lenski. 1997. Test of synergistic interactions between deleterious mutations in bacteria. Nature 390:395-398.

Ewens, W. 2004. An introduction to population genetics theory. 1 . Theoretical introduction. 2nd ed. Springer, New York.

Felsenstein, J. 1974. The evolutionary advantage of recombination. Genetics 78:737-756.

Fisher, R. 1922. On the dominance ratio. Proceedings of the Royal Society of Edinburgh 52:321-341.

$\rightarrow$ Franklin, I., and R. Frankham. 1998. How large must populations be to retain evolutionary potential? Animal Conservation 1:69-70.

García-Dorado, A., C. Lopez-Fanjul, and A. Caballero. 2004. Rates and effects of deleterious mutations and their evolutionary consequences. Pages 20-32 in A. Moya and E. Fontdevila, eds. Evolution of mol ecules and ecosystems. Oxford University Press, New York.

Gilpin, M., and M. Soulé. 1986. Conservation biology: the science of scarcity and diversity. Sinauer, Sunderland, MA.
Grimmett, G., and D. Stirzaker. 2001. Probability and random processes. 3rd ed. Oxford University Press, Oxford.

$\rightarrow$ Haag-Liautard, C., M. Dorris, X. Maside, S. Macaskill, D. Halligan, B. Charlesworth, and P. Keightley. 2007. Direct estimation of per nucleotide and genomic deleterious mutation rates in Drosophila. Nature 445:82-85.

$\rightarrow$ Hill, W., and A. Robertson. 1966. The effect of linkage on limits to artificial selection. Genetics Research 8:269-294.

$\rightarrow$ Kimura, M. 1979. Model of effectively neutral mutations in which selective constraint is incorporated. Proceedings of the National Academy of Sciences of the USA 75:1934-1937.

Lande, R. 1976. Natural selection and random genetic drift in phenotypic evolution. Evolution 30:314-334.

. 1993. Risks of population extinction from demographic and environmental stochasticity and random catastrophes. American Naturalist 142:911-927.

. 1994. Risk of population extinction from fixation of new deleterious mutations. Evolution 48:1460-1469.

Lande, R., S. Engen, and B. Saether. 2003. Stochastic population dynamics in ecology and conservation. Oxford University Press, Oxford.

Lotka, A. 1932. The growth of mixed populations: two species competing for a common food supply. Journal of the Washington Academy of Sciences 22:461-469.

Lynch, M., J. Blanchard, D. Houle, T. Kibota, S. Schultz, L. Vassilieva, and J. Willis. 1999. Perspective: spontaneous deleterious mutations. Evolution 53:645-663.

Lynch, M., J. Conery, and R. Bürger. 1995. Mutation accumulation and the extinction of small populations. American Naturalist 146: 489-518.

Lynch, M., and W. Gabriel. 1990. Mutation load and the survival of small populations. Evolution 44:1725-1737.

$\rightarrow$ Mace, G., and A. Purvis. 2008. Evolutionary biology and practical conservation: bridging a widening gap. Molecular Ecology 17:9-19.

$\rightarrow$ Motro, U., and G. Thompson. 1982. On the heterozygoty and the effective size of populations subject to size changes. Evolution 36: 1059-1066.

Ohta, T. 1973. Slightly deleterious mutant substitutions in evolution. Nature 246:96-98.

$\rightarrow$ Robert, A. 2011. Find the weakest link. A comparison between demographic, genetic and demo-genetic metapopulation extinction times. BMC Evolutionary Biology 11:260.

$\rightarrow$ Saccheri, I., M. Kuussaari, M. Kankare, P. Wikman, and W. Fortelius. 1998. Inbreeding and extinction in a butterfly metapopulation. Nature 392:491-494.

$\rightarrow$ Seddon, P., P. Soorae, and F. Launay. 2005. Taxonomic bias in reintroduction projects. Animal Conservation 8:51-58.

$\rightarrow$ Shaffer, M. 1981. Minimum population sizes for species conservation. BioScience 31:131-134.

$\rightarrow$ Spielman, D., B. Brook, and R. Frankham. 2004. Most species are not driven to extinction before genetic factors impact them. Proceedings of the National Academy of Sciences of the USA 101: 15261-15264.

Stephens, P., and W. Sutherland. 1999. Consequences of the allee effect for behavior, ecology and conservation. Trends in Ecology and Evolution 14:401-405.

Theodorou, T., H. Souan, and D. Couvet. 2009. Metapopulation persistence in fragmented landscapes: significant interactions between genetic and demographic processes. Journal of Evolutionary Biology 22:152-162. 
$\rightarrow$ Traill, L., C. Bradshaw, and B. Brook. 2007. Minimum viable population size: a meta-analysis of 30 years of published estimates. Biological Conservation 139:159-166.

Verhulst, P. 1844. Recherches mathématiques sur la loi d'accroissement de la population. Nouveaux Memoires de l'Académie Royale des Sciences et Belles-Lettres de Bruxelles 20:1-52.

Volterra, V. 1931. Variations and fluctuations of the number of in- dividuals in animal species living together. Pages 409-448 in R. N. Chapman, ed. Animal ecology. McGraw-Hill, New York. Wright, S. 1938. Size of population and breeding structure in relation to evolution. Science $87: 430-431$.

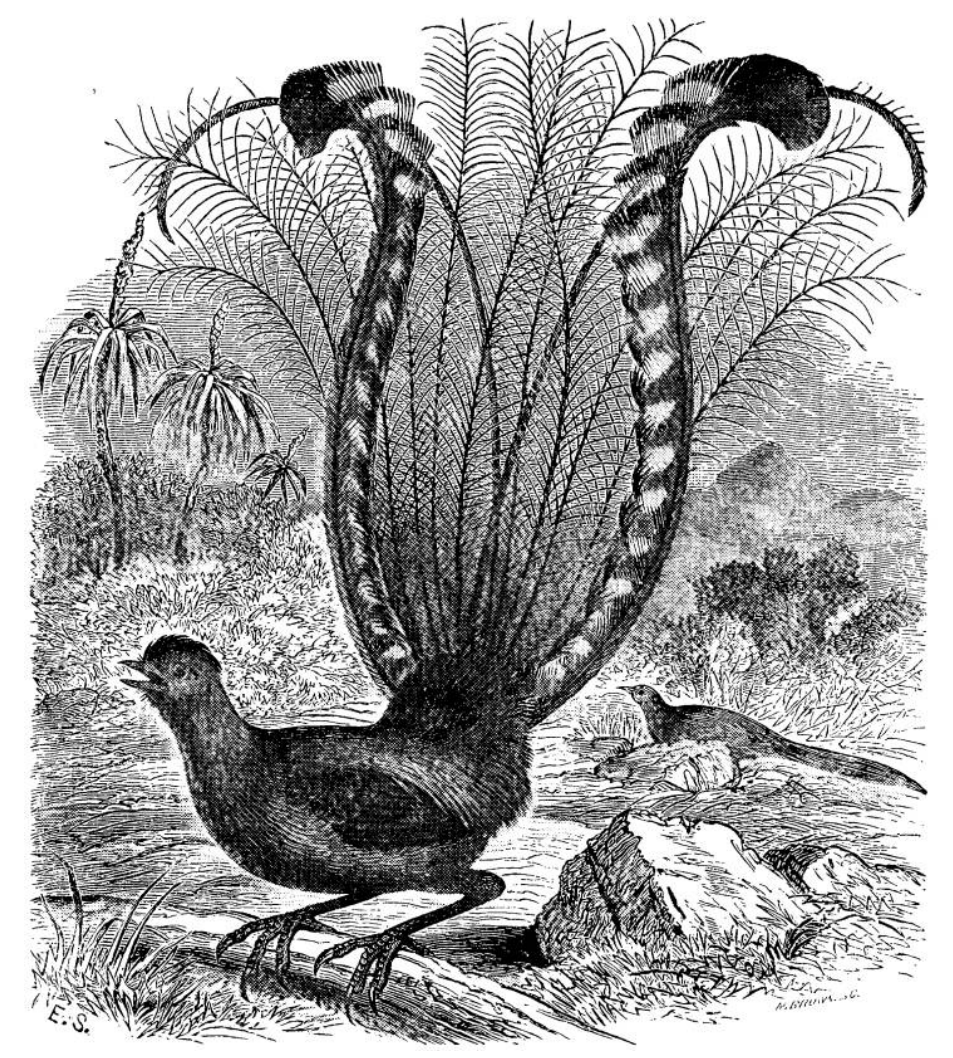

"The Lyre Bird (Menura superba) ... is extremely shy, and of all birds is the most difficult to capture, this being ascribed in part to its extraordinary powers of running and in part to the nature of the ground it inhabits, traversed as that is by immense, obstructed gullies and ravines. It seldom or never attempts to escape by flight, but ... frequently ascends trees to a considerable height, by leaping from branch to branch.” From "The Lyre Bird” by Grace Anna Lewis (American Naturalist, 1870, 4:321-326). 